ORIGINAL ARTICLE

\title{
Emergency department investigation of deep vein thrombosis
}

\author{
D A Kilroy, S Ireland, P Reid, S Goodacre, F Morris
}

Emerg Med J 2003;20:29-32

\begin{abstract}
Objectives: To apply a previously validated clinical model for predicting pre-test probability of deep vein thrombosis (DVT) to patients attending an emergency department with symptoms suggestive of DVT and assess its reproducibility in the patient population. To measure the diagnostic value of the SimpliRED D-dimer assay in effectively excluding DVT.

Method: Prospective study between March 1999 and October 2000 of adult patients attending with suspected DVT. Patients were assessed using Wells' clinical prediction tool and risk stratified. SimpliRED D-dimer estimations were then performed and compression ultrasonography arranged. The pre-test probabilities of DVT in the low, moderate, and high risk groups of Wells' original cohort were compared with the authors' figures and the sensitivity, specificity, and predictive values of the SimpliRED assay calculated for the patient population.

Results: Application of Wells' criteria to patients in the department permitted stratification into high, moderate, and low risk groups (prevalence of DVT 58.3\%, 8.9\%, and $1.5 \%$ respectively). SimpliRED D-dimer assay sensitivity was $63.4 \%$ with specificity of $74.8 \%$, with a likelihood ratio of 2.52 for a positive test and 0.49 for a negative test.

Conclusions: Clinical risk stratification allowed patients to be categorised into high, moderate, and low risk groups, albeit with less discriminatory power than originally described by Wells. The low sensitivity of the SimpliRED D-dimer assay when used routinely in a busy emergency department raises substantial doubt over the use of this test to rule out DVT, even in low risk patients.
\end{abstract}

See end of article for authors' affiliations

..................

Correspondence to: Dr D A Kilroy, Department of Emergency Medicine, Northern General Hospital, Herries Road, Sheffield S5 7AU, UK;

dakilroy@doctors.org.uk

Accepted for publication

1 March 2002
$\mathrm{T}$ he diagnosis and investigation of suspected deep vein thrombosis (DVT) has been the subject of considerable interest in recent years, stemming from the previously reported inaccuracy of clinical assessment ${ }^{1}$ and the variety of investigation protocols used in different centres. Contrast venography has been considered the gold standard for confirmation of DVT but its invasiveness and limited availability in practice make it an unattractive diagnostic tool. Compression ultrasonography is an alternative that is now widely accepted as the standard non-invasive test. Its sensitivity and specificity in detecting DVT are greater in proximal than in calf DVT, which, although in itself a condition unlikely to give rise to pulmonary embolus, may subsequently extend more proximally with the attendant risk of embolisation. ${ }^{2}$ Clinical assessment of the pretest probability of DVT has been studied and a set of validated criteria established and refined by Wells et al with the aim of reducing the amount of diagnostic testing required. ${ }^{3}$ The availability of "near patient" D-dimer assay (SimpliRED, Agen Diagnostics) has the implication that patients can be more speedily assessed and treated without the need for empirical heparinisation and admission pending a "diagnostic" test, although its sensitivity for calf DVT is only moderate. ${ }^{4}$

The purpose of our study was to validate the Wells clinical criteria in our patient population and then to determine the utility of SimpliRED D-dimer assay in a busy emergency department, performed by trained but unsupervised staff on a broad spectrum of unselected patients with suspected DVT. The investigation protocol was completed by duplex ultrasonography. We aimed to determine if using such a protocol was a safe and effective way to investigate this common problem.

\section{METHOD}

This prospective study between March 1999 and October 2000 involved 296 consenting patients over the age of 18 who presented to the Emergency Department of Northern General
Hospital, Sheffield, UK with symptoms suggestive of DVT. The investigation protocol used gained local ethical approval.

Patients were assessed against the criteria laid down in the validated clinical model for patients with suspected DVT ${ }^{5}$ and ranked accordingly into low, moderate, or high pre-test probability of having DVT (see fig 1).

Near patient D-dimer assay was then performed using the SimpliRED test by the assessing doctor. Any degree of agglutination in the test well was recorded as a positive result. All emergency department medical staff had received training in the use of the test kit. Compression ultrasonography was then arranged for all patients; if a scan was not possible the same day then the patient was either allowed home after receiving $1.5 \mathrm{mg} / \mathrm{kg}$ subcutaneous clexane empirically, or admitted overnight, based on their pre-test probability and D-dimer test result (see fig 2 ). Patients with a low pre-test probability in whom the D-dimer result was negative (no test well agglutination) and who had a negative scan were considered not to have DVT. Patients who had a positive D-dimer but negative scan, those ranked as moderate pre-test probability but having a negative D-dimer assay and those in whom the scan proved equivocal were reassessed by repeat scan one week later. Patients who were ranked as high pre-test probability and who attended out of hours were admitted and heparinised pending ultrasound scan the following day. We applied the investigation protocol to patients attending at any time of the day or night.

Ultrasound scan was performed by the Medical Physics Department of Northern General Hospital by operators unaware both of the clinical ranking of the patients and their D-dimer result. Any ultrasonographic evidence of a compression defect was taken to be a positive DVT.

Analysis of the data obtained was performed to establish whether we could demonstrate reproducibility of Wells' clinical model for determining pre-test probability of DVT and to assess the usefulness of the SimpliRED D-dimer assay in our hands. 
Clinical features (in patients with symptoms in Score both legs the more symptomatic one is used)

Active cancer treatment ongoing/within past $6 \quad 1$ months/palliative

Paralysis/paresis/recent plaster

immobilisation of lower extremities

Recently bedridden $>3$ days/major surgery within 4 weeks

\begin{tabular}{ll}
\hline Entire leg swollen & 1 \\
\hline $\begin{array}{l}\text { Calf swelling }>3 \mathrm{~cm} \text { compared with } \\
\text { asymptomatic leg } 10 \mathrm{~cm} \text { below tibial tuberosity }\end{array}$ & 1 \\
\hline Pitting oedema greater in the symptomatic leg & 1 \\
\hline Collateral superficial veins (non-varicose) & 1
\end{tabular}

Alternative diagnosis as likely as or greater than deep vein thrombosis

Total score $=$

Scoring system:

Low $=0$ or less Moderate $=1-2 \quad$ High $=3$ or more

Figure 1 Clinical assessment form for patients with suspected DVT.

\section{RESULTS}

Of the cohort of 296 patients, 279 were assessed by the full protocol. Seventeen patients did not have D-dimer estimations performed and these were excluded from statistical analysis relating to SimpliRED. The data obtained during the course of the study and the analyses performed are most easily presented in table form.

\section{Table 1}

Results of near patient SimpliRED D-dimer testing of 279 patients showing the proportions of positive and negative tests shown to have DVT or not on subsequent compression ultrasonography.

\section{Table 2}

Sensitivity, specificity, negative, and positive predictive values (NPV, PPV) and likelihood ratios for SimpliRED D-dimer test-

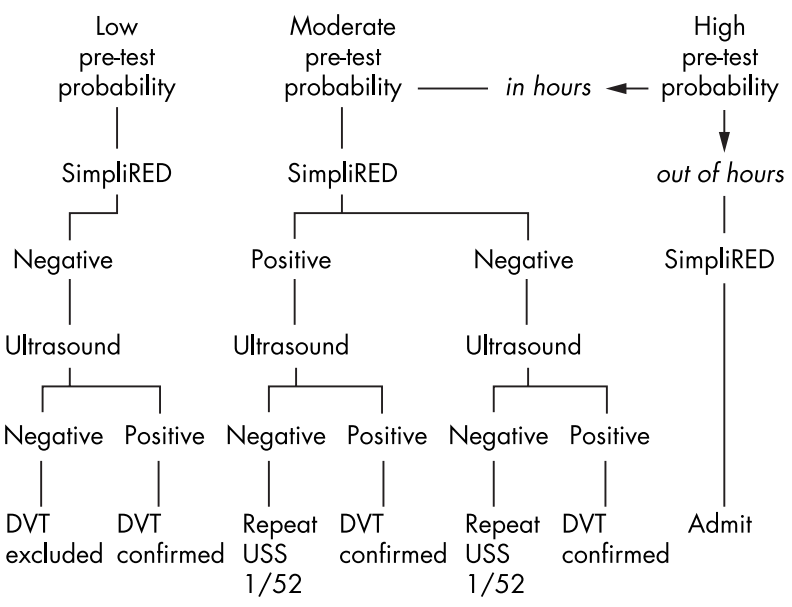

Figure 2 Investigation pathway for patients with suspected DVT.
Table 1 Results of near patient SimpliRED D-dimer testing of 279 patients showing the proportions of positive and negative tests shown to have DVT or not on subsequent compression ultrasonography

\begin{tabular}{llll}
\hline & DVT & No DVT & Total \\
\hline D dimer positive & 26 & 60 & 86 \\
D dimer negative & 15 & 178 & 193 \\
Total & 41 & 238 & 279 \\
\hline & & \\
\end{tabular}

Table 2 Sensitivity, specificity, negative, and positive predictive values (NPV, PPV) and likelihood ratios (LR) for SimpliRED D-dimer testing as performed by all grades of emergency department medical staff on adult patients presenting with suspected DVT having been assessed using the clinical prediction model

\begin{tabular}{lll}
\hline Parameter & Point estimate & $95 \% \mathrm{Cl}$ \\
\hline Sensitivity (\%) & 63.4 & 48.1 to 76.4 \\
Specificity (\%) & 74.8 & 68.9 to 79.9 \\
PPV (\%) & 30.2 & 21.5 to 40.6 \\
NPV (\%) & 92.2 & 87.6 to 95.2 \\
LR of (+) test & 2.52 & 1.83 to 3.46 \\
LR of (-) test & 0.49 & 0.32 to 0.74 \\
\hline
\end{tabular}

ing as performed by all grades of emergency department medical staff on adult patients presenting with suspected DVT having been assessed using the clinical prediction model.

Table 3

Risk stratification of our patient population using Wells' validated criteria.

Although risk stratification was successful in our group, it was not quite as discriminatory as previously described: prevalence of DVT in the low and moderate groups was significantly higher than suggested by the Wells criteria and prevalence in the high groups was significantly lower. Nevertheless, risk stratification was confirmed to be a valuable tool.

Table 4

The table shows Bayesian analysis of D-dimer results.

Wells' stratification has been used to generate pretest probabilities and pretest odds for the three clinical groups. Our likelihood ratios were then applied to these values to generate post-test odds that have been converted back into probabilities, hence giving the probability of DVT in each clinical group after the D-dimer result is known.

\section{DISCUSSION}

The fact that individual symptoms and signs have previously been reported to be inaccurate in the diagnosis of DVT has generated interest in clinical prediction models for some time. ${ }^{367}$ One of the more methodologically sound of these was that devised by Wells and colleagues in 1995 in which patients were assigned to a clinical pre-test probability (PTP) group based on "major" and "minor" clinical criteria. This model was refined by means of logistic regression analysis and prospectively re-validated to achieve discrete clinical DVT predictors. ${ }^{5}$ Application of the model to a patient cohort led to DVT prevalence of $74.6 \%$ in those ranked as "high" PTP, $16.6 \%$ of those ranked "moderate", and $3.0 \%$ of those ranked "low". Our study figures of $53.3 \%, 24.5 \%$, and $6.4 \%$ respectively demonstrate that, although risk stratification was successful, it was 
Table 3 Risk stratification of our patient population using Wells' validated criteria

\begin{tabular}{lllll}
\hline & DVT & No DVT & Prevalence $(95 \% \mathrm{CI})$ & DVT probability (Wells) \\
\hline Low risk & 12 & 175 & $6.4 \%(3.7$ to 10.9$)$ & $3.0 \%$ \\
Mod risk & 23 & 71 & $24.5 \%(16.9$ to 34.0$)$ & $16.6 \%$ \\
High risk & 8 & 7 & $53.3 \%(30.1$ to 75.2$)$ & $74.6 \%$ \\
All patients & 43 & 253 & $14.5 \%(11.0$ to 19.0$)$ & \\
\hline
\end{tabular}

Table 4 Bayesian analysis of D-dimer results

\begin{tabular}{|c|c|c|c|c|c|}
\hline \multirow{2}{*}{$\begin{array}{l}\text { Pretest probability of } \\
\text { DVT (Wells) }\end{array}$} & \multirow[b]{2}{*}{ Pretest odds } & \multicolumn{2}{|c|}{ + D-dimer post-test } & \multicolumn{2}{|c|}{ - D-dimer post-test } \\
\hline & & Odds & Probability & Odds & Probability \\
\hline Low-3.0\% & $1: 32.3$ & $1: 12.8$ & $7.2 \%$ & $1: 65.9$ & $1.5 \%$ \\
\hline Mod-16.6\% & $1: 5.0$ & $1: 2.0$ & $33.3 \%$ & $1: 10.2$ & $8.9 \%$ \\
\hline High-74.6\% & 2.9:1 & 7.3:1 & $88.0 \%$ & $1.4: 1$ & $58.3 \%$ \\
\hline
\end{tabular}

not quite as discriminatory as Wells' data described. Prevalence of DVT in our "low" and "moderate" PTP groups was significantly higher than Wells' figures while prevalence in the "high" cohort was significantly lower. Nevertheless, risk stratification is confirmed to be a valuable tool in the context of a diagnostic pathway. Even within the confines of a structured clinical assessment, observer variability in the interpretation of some clinical features may occur and our study may have benefited from two independent physicians assessing the same patient on the same visit, each blinded to the other's scoring of PTP, followed by a statistical determination of agreement. In everyday practice, however, this is clearly impractical. The important point is that PTP scoring gives clinical examination a measurable value and reduces the likelihood of inconsistent clinical assessment.

The role of SimpliRED D-dimer assay has been studied previously, with sensitivities of up to $94 \%$ quoted for its overall ability to exclude DVT $^{8}$ although elsewhere its sensitivity has been recorded at $70 \%$ for exclusion of calf DVT and recent studies have produced an even lower overall sensitivity of $65 \%$ with a negative predictive value of $81 \% .{ }^{9}$ In our patient population as a whole, D-dimer sensitivity was $63.4 \%$, which leads us to conclude that SimpliRED D-dimer assay alone is unsatisfactory as an exclusion test. The potential advantages of the assay lie in the speed and convenience with which it can be performed: because it is a quick and simple test, the sensitivity and specificity do not have to be perfect for it to add to a clinical assessment. Likelihood ratios and Bayesian analysis allow determination of whether D-dimer assay adds anything useful to clinical scoring. However, one must first decide how D-dimer might be helpful and at what threshold probability of DVT one would make certain decisions-for example, a negative D-dimer could potentially rule out DVT in a patient with a "low" clinical PTP providing the threshold for considering DVT to be ruled out lies between the pre-test and post-test probabilities. Our Bayesian analysis shows that, even using this approach, D-dimer assay is unlikely to add anything useful to the clinical assessment. In the example above, the pre-test probability for DVT in low risk patients was 3.0\% (Wells) and after a negative D-dimer it fell to $1.5 \%$ (table 4 ). Unless we consider $3.0 \%$ to be an unacceptably high risk of DVT and $1.5 \%$ to be an acceptable risk, D-dimer assay will not influence the decision to do further testing. Similar analyses can determine whether a positive D-dimer in a high risk patient might obviate the need for confirmatory testing; again, the answer would seem to be no. A "positive" SimpliRED result arises via erythrocyte agglutination in the presence of raised D-dimer levels, which can be visualised within five min- utes. Any such qualitative test has a margin of error. Although our medical staff of all grades were trained in, and monitored, performing the test, ongoing confounding may arise through poor technique (for example, failure to bring the reagents properly to room temperature and failure to correctly interpret a marginally positive result) unrelated to seniority. We made no differentiation in our data according to grade of staff as this would in practice be an unrealistic way of administering the test. Original research into the performance of SimpliRED actually stated that " . . it is possible to grade the positivity of the tes $\mathrm{t}^{\prime \prime 4}$ but no further analysis in that regard was performed and it remains unclear whether this may affect the test's reliability in practice. Our study contained a potential source of bias in that assays were done by the same doctor who had performed the PTP estimation, although the recent report quoting a SimpliRED sensitivity of $65 \%$ was achieved via a protocol where assay was done by laboratory staff, the physicians being blinded to the result. Overall, however, our results realistically reflect how SimpliRED assay performs in a routine busy emergency department setting, with limited supervision of testing procedures and naturally variable quality control.

Lower extremity ultrasonography has been widely used as a benchmark non-invasive test for DVT diagnosis ${ }^{10}$ as it is generally easy to arrange, cheap, and patient friendly. Its sensitivity in detecting calf DVT is poorer than for proximal lesions ${ }^{2}$ and there is a degree of operator variability. Its choice as the practical gold standard has arisen because contrast venography, while diagnostically superior, is invasive, time consuming, and has potentially unacceptable side effects. The ultrasound regimen is therefore well recognised in practice as the diagnostic benchmark. The main finding of our study, namely the poor diagnostic performance of SimpliRED assay, is unlikely to be influenced by any credible variation in the accuracy of ultrasound-it is not conceivable that SimpliRED actually had a sensitivity of $80 \%-90 \%$ and our results arose only because of erroneous ultrasound scanning. The overall aim of the study was to evaluate the validated clinical prediction tool and to assess the usefulness of SimpliRED estimation with the inference of incorporating them into a safe and easily applicable investigation protocol for suspected DVT. It is important to recognise the limitations of the components of any protocol in isolation and also to guard against overrarification of the investigations required to safely exclude DVT. Based on our data we cannot accept that SimpliRED D-dimer assay can safely be used in everyday practice as an exclusion tool for DVT.

In conclusion, DVT is a difficult condition to diagnose clinically. Application of the Wells criteria to patients in our 
department permitted stratification into high, moderate, and low risk groups (prevalence of DVT 58.3\%, 8.9\%, and $1.5 \%$ respectively). This is not as discriminatory as Wells' original data and may be explained by insufficient ongoing training or interobserver variation within our staff. SimpliRED D-dimer assay had a sensitivity of $63.4 \%$ and specificity of $74.8 \%$ for DVT. These findings raise doubts regarding the use of risk stratification and SimpliRED D-dimer assay to exclude DVT without further diagnostic testing within the setting of a busy emergency department.

\section{Note regarding statistical analysis}

Standard equations were used to calculate the prevalence of DVT and the sensitivity, specificity, positive, and negative predictive values and likelihood ratios for the SimpliRED D-dimer. ${ }^{11-13}$ CIA software for Windows ${ }^{13}$ was used to calculate confidence intervals using Wilson's method for proportions and the log method for likelihood ratios. Low, moderate, and high risk populations as defined by Wells' criteria were subjected to Bayesian analysis, using likelihood ratios from our data, to evaluate the potential role of $\mathrm{D}$-dimer in each population. ${ }^{11}{ }^{12}$

\section{Contributors}

Darren Kilroy wrote the paper and supervised the project; Sian Ireland and Paul Reid coordinated data collection and provided initial statistical analysis; Francis Morris initiated the study, designed the original protocol, and supervised the project; Steve Goodacre reviewed the data and provided detailed statistical analysis and comment.

\section{Authors' affiliations}

D A Kilroy, S Ireland, P Reid, S Goodacre, F Morris, Department of Emergency Medicine, Northern General Hospital, Sheffield, UK
Funding: none

Conflicts of interest: none

\section{REFERENCES}

1 Haeger K. Problems of acute deep venous thrombosis I: the interpretation of signs and symptoms. Angiology 1969;20:219-23.

2 Rose SC, Zweibel WJ, Murdoch LE. Insensitivity of color Doppler for detection of calf deep vein thrombosis. J Vasc Interv Radiol 1993:4:111-17.

3 Wells PS, Hirsh J, Anderson DR. Accuracy of clinical assessment of deep-vein thrombosis. Lancet 1995;345:1326-30.

4 Wells PS, Brill-Edwards P, Stevens P, et al. A novel and rapid whole-blood assay for D-dimer in patients with clinically suspected deep vein thrombosis. Circulation 1995:91:2184-7.

5 Wells PS, Anderson DR, Mormanis J, et al. Value of assessment of pre-test probability of deep vein thrombosis in clinical management. Lancet 1997;350:1795-8

6 Vine HS, Hillman B, Hessel SJ. Deep vein thrombosis: predictive value of signs and symptoms. Am J Roentgenol 1981;136:167-71.

7 Landefeld CS, McGuire E, Cohen AM. Clinical findings associated with acute proximal deep vein thrombosis : a basis for quantifying clinical judgment. Am J Med 1990;88:382-8.

8 Wells PS, Anderson DR, Bormanis J, et al. SimpliRED D-dimer can reduce the diagnostic tests in suspected deep vein thrombosis. Lancet 1998;351:1405-6.

9 Farrell S, Hayes T, Shaw M. A negative SimpliRED D-dimer assay result does not exclude the diagnosis of deep vein thrombosis or pulmonary embolism in emergency department patients. Ann Emerg Med 2000;35:2.

10 Perrier A, Desmarais S, Mirais M-J, et al. Non-invasive diagnosis of venous thromboembolism in outpatients. Lancet 1999;353:190-5.

11 Sackett DL, Haynes RB, Guyatt GH, et al. Clinical epidemiology: a basic science for clinical medicine. 2nd edn. Boston: Little, Brown, 1991

12 Jaeschke R, Guyatt GH, Sackett DL. Users' guide to the medical Literature III. How to use an article about a diagnostic test.B. What are the results and how will they help me in caring for my patients? The Evidence-Based Medicine Working Group. JAMA 1994;271:703-7.

13 Altman DG, Machin D, Bryant TN, et al. Statistics with confidence. 2nd edn. London: BM Books, 200. 